\title{
Dosimetry Based on ${ }^{99 m}$ Tc-Macroaggregated Albumin SPECT/CT Accurately Predicts Tumor Response and Survival in Hepatocellular Carcinoma Patients Treated with ${ }^{90}$ Y-Loaded Glass Microspheres: Preliminary Results
}

\author{
Etienne Garin ${ }^{1-3}$ Laurence Lenoir $^{1}$, Yan Rolland ${ }^{4}$, Julien Edeline ${ }^{2,5}$, Habiba Mesbah ${ }^{6}$, Sophie Laffont ${ }^{1,3}$, Philippe Porée ${ }^{6}$, \\ Bruno Clément $^{3}$, Jean-Luc Raoul ${ }^{7}$, and E. Boucher ${ }^{3,5}$ \\ ${ }^{1}$ Department of Nuclear Medicine, Comprehensive Cancer Institute Eugène Marquis, CS 44229, F-35042 Rennes, France; ${ }^{2}$ University \\ of Rennes 1, F-35043 Rennes, France; ${ }^{3}$ INSERM, U-991, Liver Metabolisms and Cancer, F-35033 Rennes, France; ${ }^{4}$ Department of \\ Medical Imaging, Comprehensive Cancer Institute Eugène Marquis, CS 44229, F-35042 Rennes, France; ${ }^{5}$ Department of Medical \\ Oncology, Comprehensive Cancer Institute Eugène Marquis, CS 44229, F-35042 Rennes, France; ${ }^{6}$ Department of Medical \\ Informatics, Comprehensive Cancer Institute Eugène Marquis, CS 44229, F-35042 Rennes, France; and ${ }^{7}$ Department of Medical \\ Oncology, Comprehensive Cancer Institute Paoli Calmette, F-13273 Marseille, France
}

Radioembolization of liver cancers using ${ }^{90}$ Y-loaded microspheres is experiencing more widespread use. However, few data are available concerning the doses delivered to the tumors and the healthy liver. This retrospective study was conducted to calculate the tumor dosimetry (planned tumor dose [ $\left.T_{\text {plan }} D\right]$ ) and nontumor dosimetry in patients treated by ${ }^{90} \mathrm{Y}$-loaded glass microspheres and determine whether tumor dosimetry could predict response and survival. Methods: Thirty-six patients with hepatocellular carcinoma (HCC), including 16 with portal vein thrombosis (PVT), were treated with ${ }^{90} \mathrm{Y}$-loaded glass microspheres. The $T_{\text {plan }} D$ and the dose delivered to the injected healthy liver were calculated using a quantitative analysis of the $99 \mathrm{~m} T \mathrm{c}$-macroaggregated albumin ( ${ }^{99 \mathrm{mT}} \mathrm{Tc}-\mathrm{MAA}$ ) SPECT/CT exam. Responses were assessed after $3 \mathrm{mo}$, using the criteria of the European Association for the Study of the Liver. Progression-free survival (PFS) and overall survival (OS) were evaluated using Kaplan-Meier tests. Results: The response rate was $69 \%$ for the overall population and $75 \%$ for the PVT patients. The dose delivered to the tumor was the only parameter associated with response with multivariate analysis $(P=0.019)$. A threshold $\mathrm{T}_{\text {plan }} \mathrm{D}$ value of $205 \mathrm{~Gy}$ was predictive of response, with a sensitivity of $100 \%$ and an accuracy of $91 \%$. Quantitative $99 m$ TcMAA SPECT/CT allowed us to increase the injected activity for 4 patients with large lesions. PFS was only 5.2 mo and OS 9 mo when using a $\mathrm{T}_{\text {plan }} \mathrm{D}$ of less than $205 \mathrm{~Gy}$ versus $14 \mathrm{mo}(P=$ $0.0003)$ and $18 \mathrm{mo}(P=0.0322)$, respectively, with a $T_{\text {plan }} \mathrm{D}$ of 205 Gy or more. Conclusion: Quantitative ${ }^{99 m}$ Tc-MAA SPECT/ CT is predictive of response, PFS, and OS. Dosimetry based on 99mTc-MAA SPECT/CT can be used for the selection of patients and for an adaptation of treatment planning, especially in selected patients (particularly in the case of large tumors). These results also confirm the efficacy and safety of ${ }^{90} \mathrm{Y}$-loaded micro-

Received Jun. 7, 2011; revision accepted Sep. 15, 2011.

For correspondence or reprints contact: Etienne Garin, Comprehensive Cancer Institute Eugène Marquis, Department of Nuclear Medicine, CS 44229, F-35042 Rennes, France.

E-mail: e.garin@rennes.fnclcc.fr

COPYRIGHT @ 2012 by the Society of Nuclear Medicine, Inc. spheres in treating HCC, even in the presence of PVT (and especially when ${ }^{99 m}$ Tc-MAA uptake is seen inside the PVT).

Key Words: radioembolization; hepatocellular carcinoma; ${ }^{90} \mathrm{Y}$, dosimetry

J Nucl Med 2012; 53:255-263

DOI: 10.2967/jnumed.111.094235

$\mathbf{H}$ epatocellular carcinoma (HCC) is a highly prevalent tumor that is difficult to treat and represents the third cause of death by cancer worldwide, with 500,000 deaths per year (1).

Among patients diagnosed with HCC, one third, classified as intermediate stage with the Barcelona Clinic liver cancer criteria, will be suitable for an intraarterial treatment, such as transarterial chemoembolization or radioembolization.

Radioembolization consists of administering a large dose of activity into the hepatic artery to deliver a high level of radioactivity to the target hepatic volume (hepatic lobe containing the tumors). ${ }^{90}$ Y-labeled microspheres are in more widespread use (2-5). Before treatment initiation, this approach requires a diagnostic angiography and a liver perfusion scan after intraarterial injection of ${ }^{99 \mathrm{~m}} \mathrm{Tc}$-macroaggregated albumin ( $\left.{ }^{99 \mathrm{~m}} \mathrm{Tc}-\mathrm{MAA}\right)$ to assess the risk of extrahepatic deposition of microspheres and evaluate the percentage of lung shunting.

Actually, there is no randomized study concerning the use of ${ }^{90}$ Y-labeled microspheres in HCC. However, several nonrandomized studies have demonstrated that glass microspheres are well tolerated, with response rates of $60 \%$ if tumoral necroses are considered (3-5). Studies concerning portal vein thrombosis (PVT) have also yielded some interesting results $(4,5)$. Several randomized studies are currently being set up or are in the recruitment stage. 
The common goal of glass microsphere radioembolization is to deliver a radiation dose of $120 \pm 20$ Gy to the injected liver volume (IL D). The calculation of this dose is based on the following accepted simplified formula:

$$
\mathrm{ILD}_{(\mathrm{Gy})}=\mathrm{IA}_{(\mathrm{GBq})} \times(1-\mathrm{S}) \times 50 / \mathrm{W}_{(\mathrm{kg})},
$$

where IA represents the activity to be injected; $S$ the percentage of pulmonary shunt, as measured by MAA liver perfusion scan; and $\mathrm{W}$ the mass of the liver volume to be treated (with $\mathrm{W}=$ volume $\times 1.03$ ). However, this simple dosimetry does not evaluate the dose absorbed by the tumor or the nontumoral liver.

To find a relationship between treatment efficacy, in terms of response and survival, and the tumor dosimetry, we conducted a retrospective study of 36 consecutive patients treated in our institution. The aim of this study was to determine whether there was a threshold tumoral dose required to obtain an objective response and to check whether this threshold was predictive of response and survival.

\section{MATERIALS AND METHODS}

\section{Patient Characteristics}

From December 2006 to June 2010, 36 consecutive patients with HCC were treated by ${ }^{90} \mathrm{Y}$-loaded glass microspheres (TheraSphere; Nordion) in a palliative setting. The use of radioembolization was approved by the ethics committee of Rennes, and written informed consent was obtained for each patient. Indication for radioembolization was determined during a multidisciplinary consultation meeting for HCC patient management. Radioembolization was the first-line treatment for 24 patients and was proposed for recurrences for 15 (5 after hepatectomy, 9 after chemoembolization, and 1 after ${ }^{131}$ I-lipiodol therapy). Patients were considered unsuitable for chemoembolization, either because of PVT or the presence of multifocal or voluminous lesions or because of incomplete response or progression after several cycles of chemoembolization. Most patients were at an intermediate or advanced stage of the disease with PVT $(n=16$ : main $=5$, lobar or segmental $=11$ ) but did not exhibit any extrahepatic spread. Table 1 reports data on the patient sample and tumor characteristics.

\section{Planning and Administration of ${ }^{90} \mathrm{Y}$-Loaded Glass Microspheres}

Microspheres were administered as described by various authors (2-6). After diagnostic angiography, a liver perfusion scan was obtained, after an injection of $185 \mathrm{MBq}$ of MAA into the hepatic artery, with planar and SPECT/CT acquisitions (window, $140 \pm 7.5 \mathrm{keV}, 32$ projections, $180^{\circ}, 128 \times 128,30 \mathrm{~s} /$ projection) (Symbia T2 gantry; Siemens). SPECT data were reconstructed using an iterative method (ordered-subset expectation maximization, 5 iterations, 8 subsets) with attenuation (low-dose CT attenuation map) and scatter correction (Jaszczak method; diffusion window, $120 \pm 7.5 \mathrm{keV}$ ) and then visualized with or without fusion with CT scan data.

A quantitative uptake analysis of tumoral and nontumoral liver tissue was performed using the Volumetric Analysis software (Syngo workstation; Siemens) as previously described (7). In the
TABLE 1

Demographic and Baseline Characteristics of Patients $(n=36)$

\begin{tabular}{|c|c|}
\hline Clinical variable & Value \\
\hline Age & $68 \pm 9(y)$ \\
\hline \multicolumn{2}{|l|}{ Sex } \\
\hline Male & 28 \\
\hline Female & 8 \\
\hline \multicolumn{2}{|l|}{ Underlying liver disease } \\
\hline Alcohol & 17 \\
\hline Hepatitis C & 8 \\
\hline Hemochromatosis & 2 \\
\hline Nonalcoholic steatohepatitis & 7 \\
\hline Biliary & 1 \\
\hline Noncirrhotic & 1 \\
\hline \multicolumn{2}{|l|}{ Child classification } \\
\hline$A$ & 30 \\
\hline B7 & 6 \\
\hline \multicolumn{2}{|l|}{ Tumor distribution } \\
\hline Unifocal & 18 \\
\hline Multifocal & 16 \\
\hline Diffuse & 2 \\
\hline Mean tumor size $\pm S D(\mathrm{~cm})$ & $7.1 \pm 5.5$ \\
\hline \multicolumn{2}{|l|}{ PVT } \\
\hline Yes & 16 \\
\hline No & 20 \\
\hline \multicolumn{2}{|l|}{ Alpha-fetoprotein level (kIU/L) } \\
\hline Mean $\pm S D$ & $6,667 \pm 17,111$ \\
\hline Median & 39 \\
\hline \multicolumn{2}{|l|}{ CLIP classification (stages $0-4$ ) } \\
\hline 0 & 8 \\
\hline 1 & 13 \\
\hline 2 & 9 \\
\hline 3 & 4 \\
\hline 4 & 2 \\
\hline \multicolumn{2}{|c|}{ Barcelona Clinic liver cancer classification } \\
\hline A & 2 (recurrences) \\
\hline B & 18 \\
\hline C & 16 \\
\hline
\end{tabular}

case of multiple treatments, the analysis was performed for the first treatment only. The accuracy (error, $<7 \%$ ) and reproducibility (interobserver concordance, 0.99 ) of this method for measuring volumes by SPECT/CT have been established by an on-phantom study (7). Briefly, this software allows semiautomatic generation of the volume of interest (VOI) in the injected liver and tumor using an isocontour method. For each VOI, the threshold value was adjusted so that the isocontours of the volume of distribution of the ${ }^{99 m}$ Tc-MAA match on the fusion images with the boundaries of the liver and tumor (Fig. 1). If the tumor was poorly delineated with the CT of the SPECT/CT, fusion was performed with a diagnostic contrast-enhanced CT. These VOIs were then used for measuring the volume of distribution of the ${ }^{99 \mathrm{~m}} \mathrm{Tc}-\mathrm{MAA}$ in the injected liver and the tumor and the total activity contained in the injected liver and the tumor. Volume and activity in the injected healthy liver were calculated by subtraction of the liver and tumor parameters.

The tumor-to-nontumor uptake ratio was also measured on SPECT/CT using a $2 \mathrm{~cm}^{3}$ region of interest positioned on the higher-uptake area of the tumor and on the surrounding healthy injected liver. 


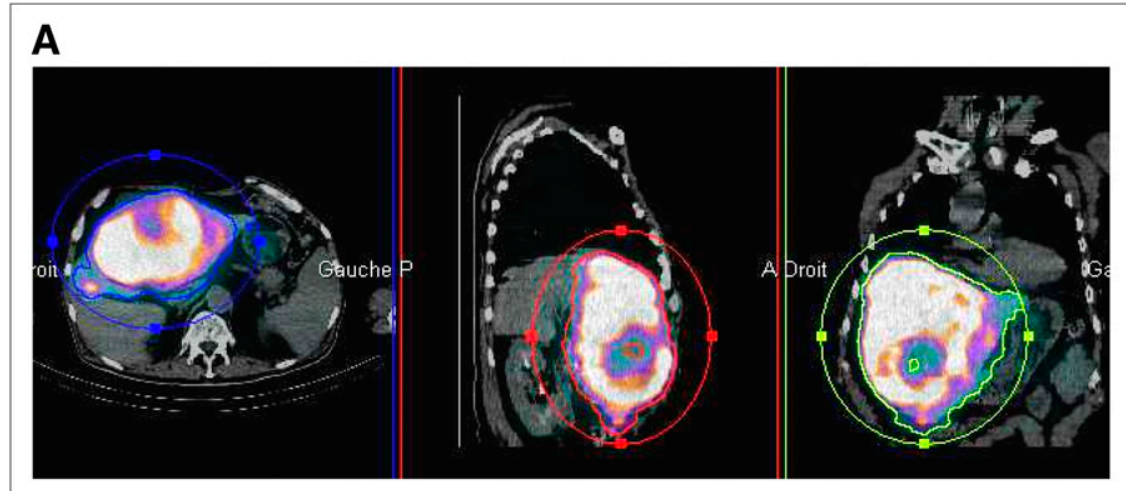

\section{B}

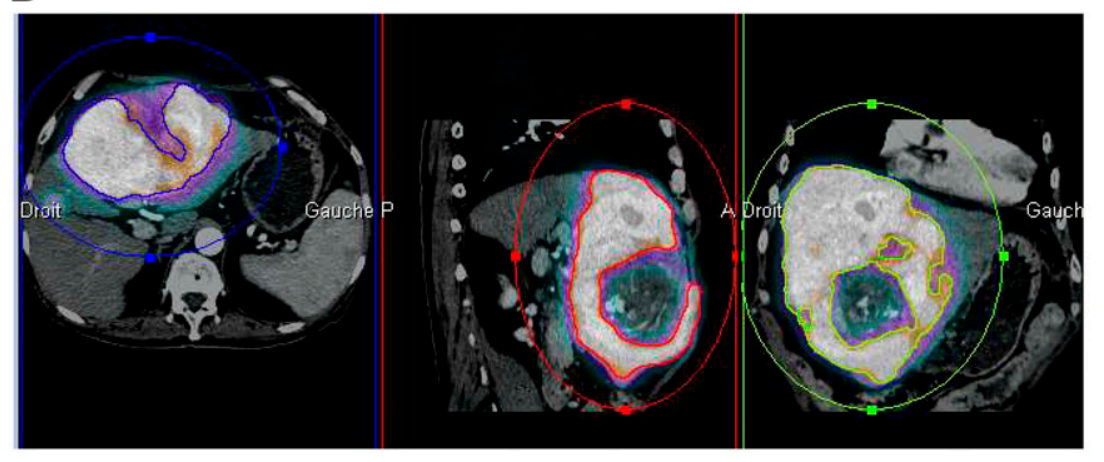

FIGURE 1. Delineation of VOls used for quantitative analysis of SPECT/CT data. VOI defined on the injected liver $(A)$ and tumor (B).
The activity to be injected was calculated conventionally for the schedule to achieve an injected liver dose of $120 \pm 20 \mathrm{~Gy}$ (without exceeding 30 Gy for the lungs). Microsphere injection was performed $8-15 \mathrm{~d}$ later. The corrected doses were also calculated after treatment administration, taking into account the activity actually injected, $\mathrm{IA}_{\text {cor }}$ (IA - residual activity remaining in tubing).

\section{Response Evaluation}

Tumor response was assessed using the criteria of the European Association for the Study of the Liver (8). Triphasic CT scans were obtained 3 mo after treatment and then every 3 mo until progression or death. Response was evaluated according to the European Association for the Study of the Liver as follows: complete response (CR), the absence of any enhancing tissue; partial response (PR), greater than $50 \%$ decrease in enhancing tissue; progressive disease (PD), an increase greater than $25 \%$ in the size of one or more measurable lesions or the appearance of new lesions; and stable disease, a tumor response between PR and PD.

\section{Statistics}

Activities are expressed in GBq, absorbed doses in Gy, and quantitative values as mean $\pm \mathrm{SD}$.

A Wilcoxon distribution-free comparison test was used to compare the doses absorbed by the tumors. The threshold dose ( $\mathrm{TD}_{\text {threshold }}$ ), that is, the tumoral dose that has to be achieved to observe a morphologic response, was determined using receiveroperating-characteristic analysis and the Youden index. To avoid missing a patient who might respond and therefore benefit from treatment, the threshold was defined as having a sensitivity of $100 \%$.

True-positives are defined as objective response (CR $+\mathrm{PR})$ obtained when tumoral dose (TD) is higher than or equal to the
$\mathrm{TD}_{\text {threshold}}$. True-negatives are defined by a TD less than $\mathrm{TD}_{\text {threshold, }}$ without an objective response at 3 mo. False-negatives are cases in which TD is less than $\mathrm{TD}_{\text {threshold }}$ and shows an objective response at 3 mo. False-positives are cases in which TD is at least as great as $\mathrm{TD}_{\text {threshold }}$ and shows no objective response at $3 \mathrm{mo}$.

Univariate analysis and a Wilcoxon distribution-free comparison test were used to identify parameters associated with tumor response, progression-free survival (PFS), and overall survival (OS). Data selected after univariate analysis were then subjected to multivariate analysis using a logistic regression test. PFS is the time between treatment and progression (CT scan) or death or second cancer. OS is the time between treatment and the last follow-up visit or death. The survival rates were estimated using the Kaplan-Meier method, and their comparison was based on a log-rank test.

We used SAS software (SAS Institute Inc.) for the statistical analyses, with a significance threshold set at a $P$ value less than 0.05 .

\section{RESULTS}

Fifty injections of ${ }^{90}$ Y-loaded glass microspheres were performed for 36 patients. Three patients did not receive any therapy ( 2 because of high hepatopulmonary shunting and 1 for failure of the angiography procedure). Fourteen patients received 2 treatments (bilateral lesions, 3; incomplete injection of the initial dose, 2 ; poor response after the first procedure, 4; and recurrence after treatment, 5). Fiftyeight lesions were assessed.

Table 2 shows the data concerning IA (planned, IA plan, and corrected, $\mathrm{IA}_{\mathrm{cor}}$ ), biodistribution, and dosimetry. The mean value of the injected healthy liver dose was $79.9 \pm$ 24.5 Gy (minimum, 19.6 Gy; maximum, 194 Gy), repre- 
TABLE 2

Biodistribution, IAs, and Dosimetry

\begin{tabular}{|c|c|c|c|}
\hline Biodistribution and dosimetric parameters & Mean $\pm S D$ & Minimum & Maximum \\
\hline Intratumoral activity (\%) & $32.8 \pm 29.4$ & 1.4 & 94.7 \\
\hline Tumor-to-nontumor uptake ratio & $7.2 \pm 7.8$ & 0.6 & 25.9 \\
\hline $\mathrm{IA}_{\text {plan }}(\mathrm{GBq})$ & $3.1 \pm 1.4$ & 1.3 & 7.5 \\
\hline $\mathrm{T}_{\text {plan }} \mathrm{D}$ (Gy) & $304.7 \pm 161.8$ & 58.4 & 872.5 \\
\hline $\mathrm{IA}_{\mathrm{cor}}(\mathrm{GBq})$ & $2.9 \pm 1.4$ & 1.2 & 7.5 \\
\hline$T_{\text {cor }} D(G y)$ & $264.4 \pm 134.5$ & 30.0 & 591.1 \\
\hline $\mathrm{IL}_{\text {cor }} \mathrm{D}(\mathrm{Gy})$ & $120.5 \pm 31.4$ & 64.3 & 197.4 \\
\hline $\mathrm{IHL}_{\text {cor }} \mathrm{D}$ (Gy) & $79.9 \pm 39.6$ & 19.6 & 166.6 \\
\hline
\end{tabular}

senting $68.3 \% \pm 32.4 \%$ (minimum, $15.3 \%$; maximum, $201 \%$ ) of the injected liver dose and was higher than $90 \%$ of the injected liver dose for 5 patients (13.8\%).

\section{Response Analysis}

Patient-Based Analysis. The 3-mo response rate was $67 \%$, with 3 patients showing CR, 21 PR, 6 stable disease, and 6 PD. The response rate was $75 \%$ for the 16 patients with PVT (12 PR, 1 stable disease, and 3 PD), whereas 6 of 12 responders had portal vein recanalization (Fig. 2) (2 with main PVT). Three PVT patients were downstaged: 1 was offered a transplantation, which he refused, and the second patient had a right hepatectomy. For the third patient, a right hepatectomy had been planned but was cancelled at the last moment after the discovery of a contralateral recurrence that had not been identified on preoperative imaging. 99m Tc-MAA uptake was seen in the PVT on the SPECT/ CT image for the 12 responding patients (Fig. 2) and only in 1 nonresponding patient with a large tumor (and a low tumor dose).

Lesion-Based Analysis. Fifty-eight tumors were analyzed. The 3-mo response rate was $72 \%$ (7 CR, $35 \mathrm{PR}$, 11 stable disease, and 5 PD). In the subgroup of patients with tumors larger than $5 \mathrm{~cm}$, the response rate was $61 \%$ (16 PR, $1 \mathrm{CR}, 7$ stable disease, and $3 \mathrm{PD}$ ) and dropped to $55 \%$ (11 PR, 5 stable disease, 4 PD) for lesions larger than $7 \mathrm{~cm}$.

Analysis of Predictive Response Factors. According to ${ }^{99 m}$ Tc-MAA-SPECT/CT analysis of the 58 tumors, the planned tumor dose $\left(\mathrm{T}_{\text {plan }} \mathrm{D}\right)$ was $372.7 \pm 142.0 \mathrm{~Gy}$ for responding lesions and $153.8 \pm 80.8 \mathrm{~Gy}$ for nonresponding lesions $(P<0.0001)$. A tumoral threshold dose value of $205 \mathrm{~Gy}$ is then required to establish an optimal dose-response ratio and avoid false-negatives. Thirteen lesions had a $\mathrm{T}_{\text {plan }} \mathrm{D}$ of less than $205 \mathrm{~Gy}$ and 45 tumors a $\mathrm{T}_{\text {plan }} \mathrm{D}$ of greater than $205 \mathrm{~Gy}$. None of the 13 lesions treated by a $\mathrm{T}_{\text {plan }} \mathrm{D}$ of less than $205 \mathrm{~Gy}$ responded to treatment. Forty of the 45 lesions treated by a $\mathrm{T}_{\text {plan }} \mathrm{D}$ of $205 \mathrm{~Gy}$ or more responded to treatment. Among the 5 nonresponding lesions, 2 received a $\mathrm{T}_{\text {cor }} \mathrm{D}$ ([Gy], tumoral corrected dose) that was far lower than the $\mathrm{T}_{\text {plan }} \mathrm{D}$ (incomplete injection of the therapeutic dose because of technical problems). The 3 other lesions treated by a $\mathrm{T}_{\text {plan }} \mathrm{D}$ of $205 \mathrm{~Gy}$ or more were large heterogeneous lesions with areas of central necrosis. None of the 13 lesions treated by a $\mathrm{T}_{\text {plan }} \mathrm{D}$ of less than 205 Gy responded to treatment.

Using a tumoral threshold dose value of 205 Gy allows us to predict the response, even before treatment is performed, with a sensitivity of $100 \%$, a specificity of $75 \%$, a negative predictive value of $100 \%$, and an accuracy of $91 \%$.

Only the $\mathrm{T}_{\text {plan }} \mathrm{D}$ and the $\mathrm{T}_{\text {cor }} \mathrm{D}$ were significantly correlated with response using univariate analysis (Table 3 ). According to multivariate analysis, only $\mathrm{T}_{\text {cor }} \mathrm{D}$ remains correlated with response $(P=0.019)$.

\section{Adapting IA According to $\mathbf{T}_{\text {plan }} \mathbf{D}$}

An interim analysis conducted after the treatment of our 20th patient identified the tumoral threshold dose value of $205 \mathrm{~Gy}$ as the response-triggering threshold. This intermediate analysis also identified 1 patient with a partly necrotic $10-\mathrm{cm}$ lesion treated with a $\mathrm{T}_{\text {plan }} \mathrm{D}$ of $237.8 \mathrm{~Gy}$ and for whom no objective response was obtained (stable disease). These provisional observations led us to increase the IA for 4 cirrhotic patients treated after the 20th case (Table 4). Three of these patients should have received, with the classic dosimetric approach, a $\mathrm{T}_{\text {plan }} \mathrm{D}$ of less than or very slightly above the threshold value of 205 Gy $(187,201$, and $209 \mathrm{~Gy}$ ). The fourth patient received a $\mathrm{T}_{\text {plan }} \mathrm{D}$ of 257 Gy, but the large $(10.6 \mathrm{~cm})$ lesion in this case was partially necrotic and associated with PVT. In all cases, we checked that a dose of less than 30 Gy was delivered to the lungs and less than 120 Gy to the healthy liver. We used a higher limit of injected healthy liver dose of $120 \mathrm{~Gy}$ as some patients have an injected healthy liver dose greater than $90 \%$ of the injected liver dose, and they can theoretically receive, using the standard (i.e., with the goal to deliver $120 \pm 20 \mathrm{~Gy}$ to the injected liver), an injected healthy liver dose reaching $128 \mathrm{~Gy}(0.9 \times 140 \mathrm{~Gy})$. The goal is to deliver $120 \pm 20 \mathrm{~Gy}$, with an injected healthy liver dose reaching $128 \mathrm{~Gy}$. One patient presented with a stabilization of the lesions, and the 3 others responded to the treatment. The patient with a 


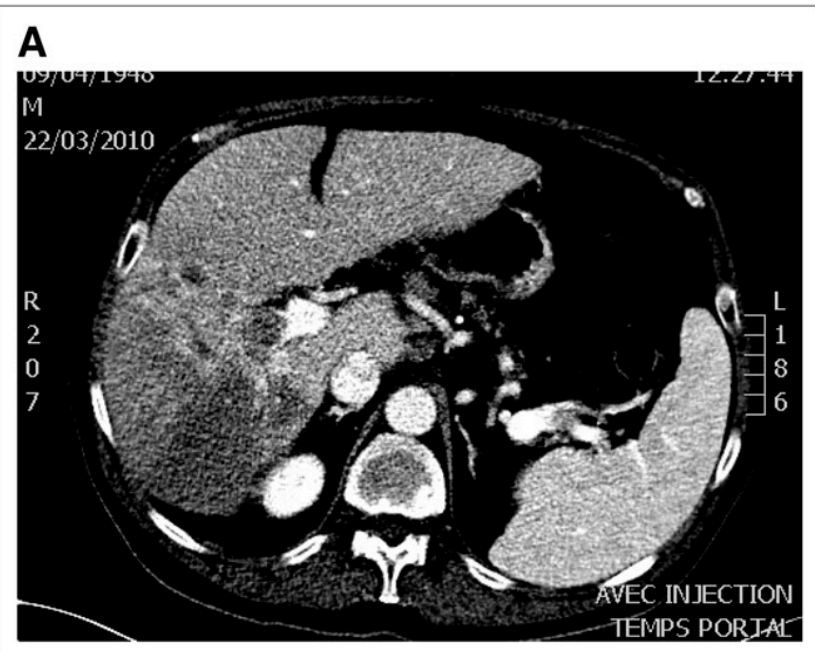

B

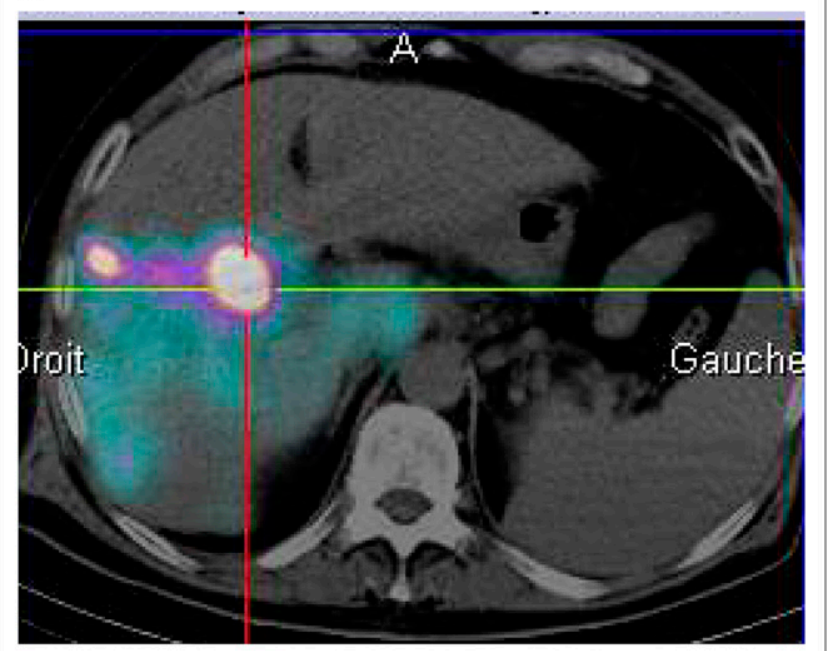

C

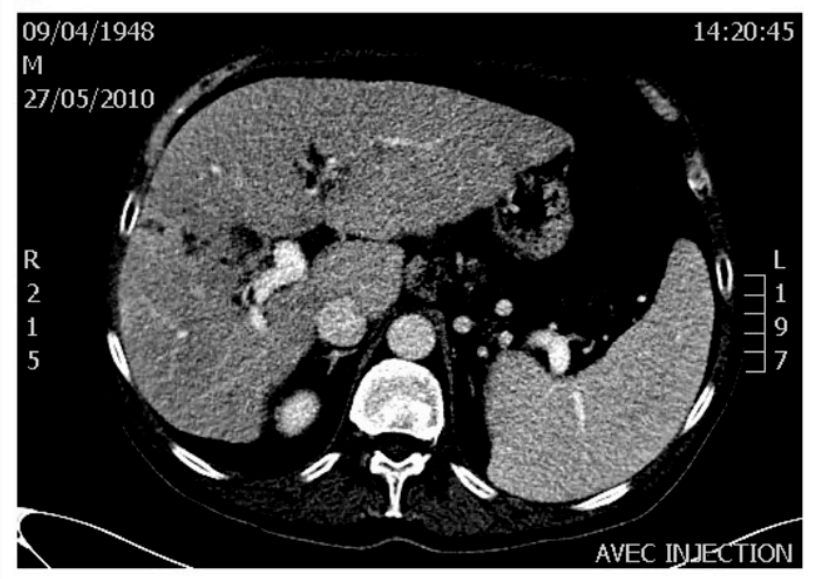

FIGURE 2. Example of major response in patient with PVT. A61-y-old patient with large HCC and PVT of right branch. (A) Initial CT slice: heterogeneous (hypervacularized and necrotic) HCC $(12.5 \mathrm{~cm})$ with PVT. (B) 99mTc-MAA SPECT/CT with high uptake in PVT. (C) CT slices 4 mo after injection of $7.5 \mathrm{GBq}$ of ${ }^{90} \mathrm{Y}$-loaded glass microspheres: major response according to the European Association for the Study of the Liver, with disappearance of tumoral hypervascularization and PVT. 10.6-cm lesion and a PVT showed a major response, including the restoration of portal venous flow. Treatment was well tolerated in all 4 patients.

Moreover, for 3 additional patients with a complex hepatic vascularization, SPECT/CT showed a whole-liver instead of a lobar perfusion, resulting in an increase of the IA. This increased IA has been dramatic in one already described case (7) $(5 \mathrm{GBq}$ instead of 0.85 initially planned).

\section{Survival Analysis}

The median follow-up was 10.5 mo (range, 6-31 mo). At the time of analysis, 15 patients had died. The 8 patients who had received treatment with a $\mathrm{T}_{\text {plan }} \mathrm{D}$ of less than 205 Gy died (6 from tumor progression and 2 from intercurrent complications unrelated to the treatment). Among the 28 patients who had received a $\mathrm{T}_{\text {plan }} \mathrm{D}$ of $205 \mathrm{~Gy}$ or more, only 7 died ( 2 because of tumor progression, 1 from liver failure, and 4 from complications unrelated to the treatment).

Overall, the median PFS was 14 mo (95\% confidence interval [CI], 10- $\infty$ mo), and OS was 18 mo (95\% CI, 10$31 \mathrm{mo}$ ). The median OS was $14 \mathrm{mo}$ (95\% CI, 9-31 mo) for patients presenting with a PVT.

Univariate analysis showed that the only parameters correlated with PFS were alpha-fetoprotein $\mathrm{T}_{\text {plan }} \mathrm{D}$, and $\mathrm{T}_{\text {cor }} \mathrm{D}$ (Table 5). In particular, the presence of a PVT was not significantly correlated with PFS (Fig. 3). According to multivariate analysis, these 3 parameters remain significantly associated with PFS $(P=0.0372$ for alpha-fetoprotein and 0.0025 for $\mathrm{T}_{\text {plan }} \mathrm{D}$ and $\left.\mathrm{T}_{\text {cor }} \mathrm{D}\right)$.

Univariate analysis showed that the only parameters correlated with OS were $\mathrm{T}_{\text {plan }} \mathrm{D}$ and $\mathrm{T}_{\text {cor }} \mathrm{D}$ (Table 5), but none remains significant according to multivariate analysis.

\section{Analysis of Survival According to Dose Absorbed by Tumor}

Median PFS was 14 mo (95\% CI, 10- $\infty$ mo) with a $\mathrm{T}_{\text {plan }} \mathrm{D}$ of 205 Gy or more and 5.2 mo $\left(95 \%\right.$ CI, 2-9 mo) with a $T_{\text {plan }} \mathrm{D}$ of less than 205 Gy $(P=0.0003)$ (Fig. 4).

Median OS was 18 mo $(95 \% \mathrm{CI}, 11-\infty \mathrm{mo})$ with a $\mathrm{T}_{\text {plan }} \mathrm{D}$ of 205 Gy or more, whereas it was only 9 mo (95\% CI, 2-31 mo) with a $\mathrm{T}_{\text {plan }} \mathrm{D}$ or less than $205 \mathrm{~Gy}(P=0.0322)$ (Fig. 4).

\section{Global Tolerance and Hepatic Toxicity}

No deaths were reported within 1 mo of treatment. Discrete to moderate asthenia was frequently reported, with much rarer occurrence of pain. Seven patients developed clinical signs of hepatic decompensation. This condition proved fatal in 1 patient with biliary cirrhosis (the patient died 2 mo after treatment) but regressed in the 6 other patients (4 were Child B status at baseline). The injected healthy liver doses delivered during treatment were 47.1, 81.5, 92.4, 114.5, 117, and 127 Gy (it could not be evaluated in 1 case of multifocal HCC).

One patient presented with septicemia, which was traced to a transduction after diagnostic angiography. 
TABLE 3

Lesion-Based Analysis: Factors (Mean \pm SD) Associated with Tumoral Response (with Univariate Analysis)

\begin{tabular}{|c|c|c|c|}
\hline Tested variables & Responding lesions & Nonresponding lesions & $P$ \\
\hline $\mathrm{IA}_{\text {plan }}(\mathrm{GBq})$ & $2.9 \pm 1.5$ & $3.3 \pm 1.2$ & Not significant \\
\hline $\mathrm{IA}_{\mathrm{cor}}(\mathrm{GBq})$ & $2.8 \pm 1.5$ & $3.0 \pm 1.3$ & Not significant \\
\hline $\mathrm{IL}_{\text {plan }} \mathrm{D}(\mathrm{Gy})$ & $126.1 \pm 31.6$ & $107.9 \pm 28.2$ & Not significant \\
\hline $\mathrm{IHL}_{\text {plan }} \mathrm{D}$ (Gy) & $78.2 \pm 32.7$ & $83.7 \pm 53.1$ & Not significant \\
\hline $\mathrm{T}_{\text {plan }} \mathrm{D}(\mathrm{Gy})$ & $372.7 \pm 142.0$ & $153.8 \pm 80.8$ & $<0.0001$ \\
\hline $\mathrm{T}_{\text {cor }} \mathrm{D}(\mathrm{Gy})$ & $327.7 \pm 106.8$ & $123.6 \pm 63.2$ & $<0.0001$ \\
\hline Tumor-to-nontumor uptake ratio & $8.3 \pm 8.9$ & $4.5 \pm 3.1$ & $<0.01$ \\
\hline Tumoral activity (\% IA) & $31.2 \pm 26.4$ & $36.6 \pm 35.9$ & Not significant \\
\hline Alpha-fetoprotein (kIU/L) & $5,068.3 \pm 18,267.1$ & $44,209.2 \pm 150,849.7$ & Not significant \\
\hline Size $(\mathrm{cm})$ & $6.7 \pm 5.9$ & $8.3 \pm 4.1$ & Not significant \\
\hline
\end{tabular}

\section{DISCUSSION}

This study yields 3 major results. First, we have found a strong correlation between response and the dose absorbed by the tumor. Before treatment initiation, the response can be predicted when using a tumoral threshold dose of 205 Gy or more, with a sensitivity of $100 \%$ and an accuracy of $91 \%$. Three false-positive results were encountered in cases involving large and heterogeneous lesions with areas of necrosis and central hypofixation, suggesting that the treatment of such lesions might benefit from an increase of the IAs. Two factors may explain these falsepositive results. First, when lesions are highly heterogeneous, the calculated tumor dose (which is averaged over the entire tumor volume) might not accurately reflect in situ conditions, with tumor areas receiving high radiation doses and other areas less than the threshold value. Second, these lesions might exhibit some degree of radioresistance, possibly related to a hypoxic state.

The fact that the tumoral planning dose value is predictive of response to radioembolization represents a major breakthrough, because no predictive factor has been yet identified. Even the recent study by Sato et al. failed to establish a correlation between lesion vascularization and survival (9). A team from Milan (Italy) has recently published a study establishing a correlation between delivered tumor dose and efficacy, backing up our results and the importance of precise dosimetric evaluation using quantitative ${ }^{99 \mathrm{~m} T c-M A A}$ SPECT/CT (10).

The second major result of our study is that the tumoral planning dose is known before treatment administration.

TABLE 4

Standard and Optimized Therapeutic Plans and Follow-up for 4 Patients with Optimization of IA According to $T_{\text {plan }} D$

\begin{tabular}{|c|c|c|c|c|}
\hline Clinical and dosimetric variables & Patient 1 & Patient 2 & Patient 3 & Patient 4 \\
\hline \multicolumn{5}{|l|}{ Tumor characteristic } \\
\hline Size & 14.7 & 8.3 & 10.6 & 12 \\
\hline No. of lesions & 1 & 4 & 1 & 1 \\
\hline PVT & Branch & No & Branch & Branch \\
\hline Child-Pugh & B7 & A5 & A6 & A5 \\
\hline \multicolumn{5}{|l|}{ Standard plan } \\
\hline $\mathrm{IA}_{\text {plan }}(\mathrm{GBq})$ & 2.5 & 2.5 & 5 & 5 \\
\hline $\mathrm{T}_{\text {plan }} \mathrm{D}$ (Gy) & 187 & $177,216,297,426$ & 257 & 201 \\
\hline IL plan D (Gy) & 148 & 110 & 131 & 106 \\
\hline $\mathrm{IHL}_{\text {plan }} \mathrm{D}(\mathrm{Gy})$ & 77 & 66 & 66 & 44 \\
\hline \multicolumn{5}{|l|}{ Optimized plan } \\
\hline $\mathrm{I}_{\text {plan }}(\mathrm{GBq})$ & 3.5 & 3.5 & 7.5 & 6.5 \\
\hline $\mathrm{T}_{\text {plan }} \mathrm{D}(\mathrm{Gy})$ & 229 & $250,305,419,601$ & 349 & 265 \\
\hline $\mathrm{IL}_{\text {plan }} \mathrm{D}(\mathrm{Gy})$ & 175 & 154 & 196 & 138 \\
\hline $\mathrm{IHL}_{\text {plan }} \mathrm{D}(\mathrm{Gy})$ & 94 & 93 & 92 & 58 \\
\hline \multicolumn{5}{|l|}{ Follow-up } \\
\hline Duration (mo) & 10 & 12 & 15 & 11 \\
\hline Response & Stable disease & PR & PR & PR \\
\hline Liver toxicity & No & No & No & No \\
\hline
\end{tabular}


TABLE 5

Factors Associated with PFS and OS (with Univariate Analysis)

\begin{tabular}{lcc}
\hline \multicolumn{1}{c}{ Variables tested } & PFS & OS \\
\hline Type (unifocal vs. multifocal and diffuse) & Not significant & Not significant \\
Size ( $\leq 5$ vs. $>5 \mathrm{~cm})$ & Not significant & Not significant \\
PVT (present) & Not significant & Not significant \\
Alpha-fetoprotein level $(\leq 400$ vs. $>400)$ & 0.009 & Not significant \\
Child-Pugh (A5 vs. A6 + B7) & Not significant & Not significant \\
CLIP (stage 0,1, and 2 vs. 3 and 4$)$ & Not significant & Not significant \\
$T_{\text {plan }} D(<205$ vs. $\geq 205$ Gy) & 0.0002 & 0.0409 \\
$T_{\text {cor }} D(<194$ vs. $\geq 194$ Gy) & 0.0002 & 0.0409 \\
\hline
\end{tabular}

Thus, the treatment schedule can be modified and adapted, either leading to the injection of higher-activity microspheres if the tumoral planning dose is less than the tumoral threshold dose or contraindicating this treatment if the optimized tumoral planning dose cannot reach the threshold dose without exceeding a dose of 30 Gy to the lungs and 120 Gy to the healthy liver. In our study, by taking into account the dose delivered both to the tumor and to the adjacent nontumoral liver, we increased significantly the activity injected in 4 of the 16 patients (with large lesions) who were treated after an interim analysis of the results. Treatment after interim analysis is preferable to optimize the activity to be injected and exceed the tumoral threshold dose at the first treatment rather than perform several treatments in succession. Indeed, with radioembolization, the absorbed dose is directly dependent on the biodistribution of the agent used (whereas, with external-beam therapy, it depends solely on the physical parameters). We have previously demonstrated with ${ }^{131}$ I-lipiodol that the dose absorbed by the tumor is more favorable after an initial treatment $(248 \pm 176 \mathrm{~Gy})$ than after a second treatment (152 \pm $122 \mathrm{~Gy})$, suggesting that the accumulation of intratumoral therapeutic agent decreases under the effect of previous treatments because of a redistribution of the therapeutic agent from the tumor into the nontumoral liver (11).

The third major finding of our study is the identification of a relationship between the dose absorbed by the tumor and survival. This finding has never before been encountered with radioembolization therapy. Disease progression can be predicted using a tumoral threshold dose higher than $205 \mathrm{~Gy}$, because these threshold values enable the identification of a group of patients with significantly higher PFS and OS. This confirms the need to reach a threshold tumor absorbed dose to achieve a good efficacy. Indeed, when the dose delivered to the tumor is equal to or higher than this threshold value, the median PFS rises from 5.2 to 14 mo $(P=0.0003)$ and OS from 9 to $18 \mathrm{mo}(P=0.0322)$.

Because there are evidently other parameters that are recognized as influencing survival (e.g., liver function, tumor burden, Barcelona Clinic liver cancer stage, comorbidity related to cirrhosis), the correlation between tumoral dose and OS (especially long-term survival) needs to be confirmed with a larger follow-up and other studies.
Liver tolerance was acceptable, with alteration of liver functions observed in 7 patients, who were all treated in a standard way - that is, without optimization of the IA as a function of the tumor dosimetry (none of the 4 patients with optimization of the IA presented with hepatic toxicity). Liver dysfunction was transient in 6 patients and fatal in only 1 patient (the patient presented with cirrhosis of biliary origin, an etiology that possibly explains the lower tolerance to irradiation). For 3 of them, the injected healthy liver dose delivered during treatment was lower than 100 Gy and even lower than 50 Gy in 1 patient. Furthermore, 5 patients treated in a standard way (i.e., 15.6\%) received doses higher than $100 \mathrm{~Gy}$ in their healthy liver tissue with no side effect $(100.5,102.2,129.2,139.8$, and $167 \mathrm{~Gy})$. These elevated values of injected healthy liver dose are found in cases of small-sized or large and highly necrotic tumors (and reached 201\% of the injected liver dose [i.e., corresponding to $167 \mathrm{~Gy}$ ] for 1 patient without any problem of toxicity), thus illustrating the difficulty of accurately determining maximum-tolerated dose for the healthy injected liver in this patient population presenting with underlying cirrhosis.

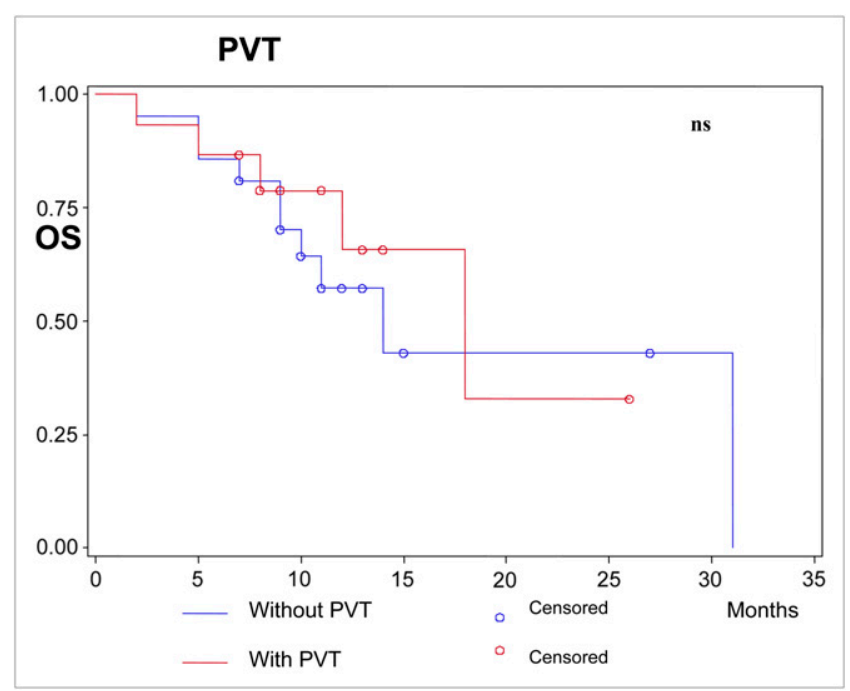

FIGURE 3. Kaplan-Meier estimates of OS stratified by portal vein status. 


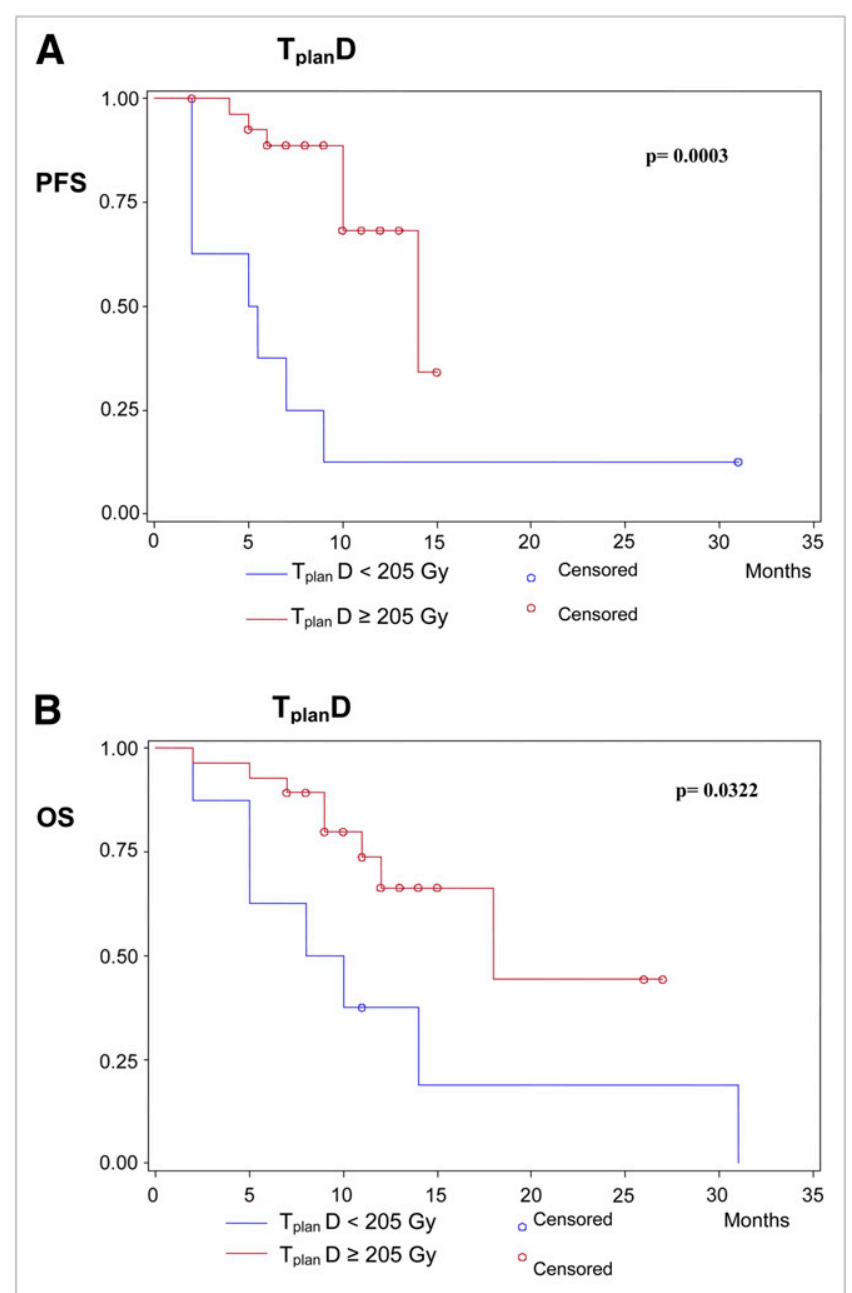

FIGURE 4. Kaplan-Meier estimates of survival stratified by tumoral dose: PFS (A) and OS (B).

Additional research is required to refine dosimetry of nontumoral liver tissue exposed to radiation. An absorbed dose escalation study could also be performed on the nontumorous liver, but it would be difficult to implement because it would first be necessary to identify clearly the various factors likely to have an influence on hepatic tolerance so the analysis could be stratified according to these various factors. However, it is difficult to identify these various factors and their weightings because of their multiplicity and the scarcity of hepatic complications. A scoring system for liver toxicity that took into account all parameters likely involved in the hepatic tolerance of radioembolization, such as morphologic (e.g., tumor burden), biologic (e.g., bilirubin level), dosimetric (injected healthy liver dose, volume of nonirradiated liver), and possibly others (etiology of cirrhosis, severity of fibrosis), and took into account different weightings for each parameter should be defined.

Regarding the efficacy of ${ }^{90} \mathrm{Y}$-loaded glass microspheres, our study confirms the good results regarding response and survival reported by other authors, even in the presence of PVT $(4,5)$.

In our study, three patients with PVT (25\%) were downstaged, one underwent a curative hepatectomy, and another was proposed for transplantation. This point is crucial, because the combination of HCC and PVT is usually considered incurable. Therefore, microspheres open the way to a possibly curative approach for certain patients. Such a possibility has recently been highlighted in a PVT patient who was able to receive a liver transplant after radioembolization (12). Moreover, in our study, the presence of PVT was not found to be significantly associated with survival in radioembolization-treated patients, suggesting that radioembolization could indeed improve the prognosis for PVT patients. The postradioembolization outcomes also compare well with sorafenib treatment $(13,14)$.

However, these preliminary results need to be confirmed by randomized studies of greater scope because of the retrospective character of this trial, the relatively restricted patient sample and the rather short duration of follow-up for certain patients. In addition, as discussed previously, further work should be performed on evaluating the hepatic tolerance to irradiation. Concerning the technical aspects, the dosimetric model used in this study is simple and is able to yield only an evaluation of the mean dose. Such an approach can be insufficient for heterogeneous lesions, especially when the evaluation of the injected healthy liver dose does not take into account irradiation via the tumor of the healthy peritumoral liver, an effect that can be important in the case of large tumors with enhanced uptake. A voxel-based dosimetry model, as previously described (10), should lead to progress in this field.

\section{CONCLUSION}

This study, for the first time, to our knowledge, demonstrates the predictive value of quantitative ${ }^{99 \mathrm{~m} T c-M A A}$ SPECT/CT regarding response to radioembolization, PFS, and OS. The use of tumor dosimetry based on ${ }^{99 \mathrm{~m}} \mathrm{Tc}-\mathrm{MAA}$ SPECT/CT data obtained after the diagnostic angiography could allow an adaptation of the therapeutic planning and the activity to be administered, especially in the case of large lesions. Our results also confirm the efficacy and safety of ${ }^{90}$ Y-loaded microspheres in treating HCC, even in the presence of PVT. Randomized studies need to be conducted to confirm these results and define the role of radioembolization in the therapeutic management of HCC, whether or not involving patients with PVT.

\section{DISCLOSURE STATEMENT}

The costs of publication of this article were defrayed in part by the payment of page charges. Therefore, and solely to indicate this fact, this article is hereby marked "advertisement" in accordance with 18 USC section 1734. 


\section{ACKNOWLEDGMENTS}

Etienne Garin is a consultant for Nordion. No other potential conflict of interest relevant to this article was reported.

\section{REFERENCES}

1. El-Serag HB. Mason AC. Rising incidence of hepatocellular carcinoma in the United States. N Engl J Med. 1999;340:745-750.

2. Salem R, Thurston KG, Carr BI, et al. Yttrium-90 microspheres: radiation therapy for unresectable liver cancer. J Vasc Interv Radiol. 2002;13:S223-S229.

3. Lewandowski RJ, Kulik LM, Riaz A, et al. A comparative analysis of transarterial downstaging for hepatocellular carcinoma: chemoembolization versus radioembolization. Am J Transplant. 2009;9:1920-1928.

4. Salem R, Lewandowski RJ, Mulcahy MF, et al. Radioembolization for hepatocellular carcinoma using yttrium-90 microspheres: a comprehensive report of long-term outcomes. Gastroenterology. 2010;138:52-64.

5. Hilgard P, Hamami M, Fouly AE, et al. Radioembolization with yttrium-90 glass microspheres in hepatocellular carcinoma: European experience on safety and long-term survival. Hepatology. 2010;52:1741-1749.

6. Salem R, Lewandowski RJ, Gates VL, et al. Technology Assessment Committee; Interventional Oncology Task Force of the Society of Interventional Radiology. Research reporting standards for radioembolization of hepatic malignancies. J Vasc Interv Radiol. 2011;22:265-278.
7. Garin E, Rolland Y, Lenoir L, et al. Utility of quantitative Tc-MAA SPECT/CT for yttrium-labelled microsphere treatment planning: calculating vascularized hepatic volume and dosimetric approach. Int J Mol Imaging. July 28, 2011 [Epub ahead of print].

8. Forner A, Ayuso C, Varela M, et al. Evaluation of tumor response after locoregional therapies in hepatocellular carcinoma: are response evaluation criteria in solid tumors reliable? Cancer. 2009;115:616-623.

9. Sato KT, Omary RA, Takehana C, et al. The role of tumor vascularity in predicting survival after yttrium-90 radioembolization for liver metastases. $J$ Vasc Interv Radiol. 2009;20:1564-1569.

10. Chiesa C, Maccauro M, Romito R, et al. Need, feasibility and convenience of dosimetric treatment planning in liver selective internal radiation therapy with ${ }^{90} \mathrm{Y}$ microspheres: the experience of the National Tumor Institute of Milan. $Q J$ Nucl Med Mol Imaging. 2011;55:168-197.

11. Becker S, Laffont S, Rolland Y, et al. Dosimetric evaluation and therapeutic response to vectorized internal radiation therapy of hepatocarcinomas using iodine-131 labelled lipiodol. Nucl Med Commun. 2008;29:815-825.

12. Ettorre GM, Santoro R, Puoti C, et al. Short-term follow-up of radioembolization with yttrium-90 microspheres before liver transplantation: new perspectives in advanced hepatocellular carcinoma. Transplantation. 2010;90:930-931.

13. Cheng AL, Kang YK, Chen Z, et al. Efficacy and safety of sorafenib in patients in the Asia-Pacific region with advanced hepatocellular carcinoma: a phase III randomised, double-blind, placebo-controlled trial. 2009;10:25-34.

14. Llovet JM, Ricci S, Mazzaferro V, et al. Sorafenib in advanced hepatocellular carcinoma. N Engl J Med. 2008;359:378-390. 